\title{
Implicated in the Indicator Game: a Response to the Debate
}

\author{
MAUREEN MCNEIL \\ LANCASTER UNIVERSITY
}

Let me begin by saying how much I welcome this collection. It is to be celebrated, as Alan Irwin (2017) encourages us to do, as exemplifying the strengths of STS as a field and the resourcefulness of its community. It is refreshing to find scholars reflecting on the structures and operations of the academic world and on their own practices, trying to develop strategies in the face of the growing pressures and consequences of the "indicator game." What makes this set of pieces distinctive in a growing literature on these developments (much of which is deployed and referenced in this issue) is that the authors try to think through what STS as a field and as a set of practices with a diverse theoretical repertoire might have to offer in the struggles to live with and against these developments. Their STS thinking is articulated with direct reference to specificities of the authors' own experiences. They offer situated reflections about doing STS and using it in dealing with "the culture of competition and quantification" (Müller 2017) which increasingly pervades academic life.

My job as I understand it is to respond to these initiatives. I have chosen to do this, in the positive spirit advocated by many of the contributors (especially, but not only, Irwin 2017), highlighting what I consider to be key features of the collection. In so doing I will also make my own suggestions about further challenges for STSers (a borrowed neologism) and possibly others as we navigate this difficult terrain.

\section{Being Implicated / Implicated Beings}

The editors' epitaph (Fochler and de Rijcke 2017, 22) for the collection is striking: a quotation from Marilyn Strathern cautioning that, in dealing with the indicator game, "we are witnessing an effect that we (practitioners in higher education) have helped produce. Auditors are not aliens: they are a version of ourselves" (Strathern 1997, 319.) Strathern's "auditors-R-us" caution haunts the project and a number of the authors mull it over. The collection is up-front in acknowledging that STSers and other academics not only get caught up in the indicator mechanisms, but turn (even sometimes are) their cogs-enabling them to run.

Some contributors flesh out how enrolments in these systems are solicited and ensured emotionally and psychically. There are allusions to the anxieties which prompt compliance, in

${ }^{1}$ Maureen McNeil, Email: m.mcneil@lancaster.ac.uk

Copyright (C) 2017 (Maureen McNeil). Licensed under the Creative Commons Attribution Non-commercial No Derivatives (by-nc-nd). Available at estsjournal.org. 
the wake of "institutional nudging and...coercion" (Fochler and de Rijcke 2017, 29). But the authors are also often quite open about their more positive emotions. Ulrike Felt (2017), for example, explores "the moments of empowerment and pleasure" enjoyed by her and others resulting from apparent successes in playing the appraisal game (see also Bal 2017; Irwin 2017; Wouters 2017). There are even references to some finding "joy in the indicator game" (Fochler and de Rijcke 2017, 23, 30). It is, perhaps, not surprising that few of us are immune to the buzz associated with being heralded as good at our jobs, or, given the investments required, that hitting metric targets or even jackpots can provide adrenalin rushes, as well as palpable rewards. Ulrike (2017) likens the operation of academic metric regimes to "the quantified-self" health movement in that both revolve around self-regulation which provides a palpable, if spurious, sense of incremental control and progress.

STS theoretical sensitivities are particularly attuned to implicatedness (if you will pardon the neologism). Notions of social constructionism, co-production, actor network theory, and intra-action all explore how this works. Moreover, neoliberal regimes and their derivative manifestations in versions of "the quantified self" are particularly insidious in their capacities to get under our skins and into our heads / psyches. Ulrike (Felt 2017, 61) refers to a variety of forms of internalization including the "new metric imagination." Yet, as many of the authors in this collection remind us, STS has been profoundly concerned with the politics of knowledge production and, over the years, STS has shown itself to be adept in tracing its workings and contesting consequential injustices. These somewhat countervailing orientations make developing strategies for resistance and contestation in dealing with the indicator game a very tricky business for STSers. I will return to strategies towards the end of my commentary, but for now I want to identify what I see as the prime tacks taken by the contributors to this collection:

- $\quad$ offering "survival narratives"
- $\quad$ tracing the logics of the indicator regime
- $\quad$ exploring the specific features of STS
tackling the publishing juggernaut

These are not totally discrete approaches so there is some overlap and considerable intertextuality within the issue. Nevertheless, it is interesting to consider the different registers of the contributions. In the following discussion I will briefly examine each of these, highlighting aspects of these interventions and offering suggestions about possible developments and challenges associated with them.

\section{Survival Narratives: STS Case Studies}

Turning to the more externalized and collective forms of implication-there are a number of references in this issue to "work-arounds"-itself a fascinating term-which in this context indicates more or less ingenuous ways of escaping some elements or consequences of accountability regimes, without upsetting the systems or disturbing their hold. There is 
equivocation about these in this issue: as they are sometimes treated as inevitable and/or accounts of these are offered as instances of reasonable or even good collective practice, and, in other cases, concern is expressed that they are inadequate, that they merely paper over the cracks, or, worse, that they induce complacency, bolster the systems, and thereby entrench the status quo.

While there are equivocations about work-arounds here, all the contributors advocate some form of contestation. Moreover, there are anecdotes within some of the essays (Felt 2017; Irwin 2017) and two articles in particular-by Roland Bal and Paul Wouters (Bal 2017; Wouters 2017) which constitute what might be called "survival narratives": participants' stories of institutional units which (at least to the point registered by the authors) have weathered the slings and arrows of sometimes outrageous appraisal. These two contributions comprise, as Roland notes, "auto-ethnographic" case studies and, in their rather small-scale versions, they could be linked to the lauded STS tradition of ethnographic laboratory studies.

Roland's and Paul's offerings are salutary tales for many reasons, not least because they are stories of success-at least of some sort-in the face of apparent adversity. It is noticeable that both entail insightful recountings of mixtures of compliance and contestation. Roland (2017) is explicit about this--as he categorizes his unit's practices under such distinct labels as "changing," "adjusting to," and "ignoring" - the evaluative regime to which it was subjected. His take-away message that performance management regimes and research practices are coconstituted was simultaneously reassuring and worrying. It made me wonder about whether practices can be as clearly delineated as his labeling suggests.

Paul urged his team, and by implication other STS practitioners, to use appraisal situations as opportunities to explore "who one wants to be." This could be a powerful reorientation. Nevertheless, there are also some curious lacunae and unacknowledged ironies embedded in the story he tells about his own group's attempt to do this. For example, despite his insistence on the performative nature of appraisal reviews, Paul is not reflective about the irony in his attribution of the relative success of his team to their "authenticity" and "sincerity" in their performances for the review panel. In addition, I was surprised that he did not consider how his group's expertise and reputation specifically as bibliometric experts might have contributed to their confidence and influenced their assessors, as they challenged the modes of their assessment. He does not reflect about how established expertise and confidence play out in dealing with regulators.

Don't get me wrong: we need narratives of this kind. Roland and Paul provide valuable on-the-ground accounts of collective dealings with appraisal procedures, fleshing out what seems to work in what circumstances and pondering gains and losses. My point is that these stories, while reassuring in some ways, belie containment as "survival narratives" given the complexity of strategies for and observations about such processes. Together these two contributions (Bal 2017; Wouters 2017) are likely to generate reflections about patterns of compliance and resistance and about the relations within collectives that may shift orientations in either direction (indeed, how we discern these directions). These accounts also raise questions, with due attention to 
situational specificity (which virtually all these authors advocate) about where, when, and how to resist and contest indicator regimes.

\section{Tracing the Logics of the Indicator Regime}

Although stories about personal experiences of assessment procedures pepper all the articles, other authors take a rather different tack. Both Alan Irwin and Ulrike Felt challenge us to move beyond fixations with the indicator game per se. In effect, they ask us to stand back and look more carefully at its logics.

Alan Irwin (2017) insistently reminds us that "indicators themselves do not have effects." The great strength of Alan's sharp review is that it disabuses nostalgic, romantic thinking and provides insights that could help to separate the wheat from the chaff. He really does offer words of wisdom (garnered through considerable, varied academic experience) which can be useful in orientating struggles about assessment and ranking including in decision making about what fights are worth having. His approach is straightforward, as he seeks to clarify the issues bubbling-up in institutionalized appraisals that should not be ignored, including: the public accountability of academia, internationalization of the academic labor market, standards of quality, and professional and existential (in)security.

For Alan it is crucial that we attempt to understand "the wider institutional and professional logic" in which "indicatorification" (his term) is embedded and to decipher what it is about, what it is (and isn't) tackling. The bulk of his article is devoted to an exploration of what he designates as the "set of questions" which animate and sustain indicator regimes. But what he outlines are not really questions, rather, they are parameters and points of address that constitute the context for these regimes. What he is offering is an account of the conditions of possibility which render these regimes, to varying degrees, plausible and effective.

I emphasize the preceding clarification not to be pedantic but because it may help to illuminate the openings afforded by Alan's essay. In passing, he asserts that indicator mania is not always hegemonic and, extracting more explicitly from his account (and supplementing it with insights from others), we can see that it can also be relatively ineffective. The key here is identifying within the evolving regimes of evaluation what merits STS community concern, as well as, within established regimes, the manifestations of inadequacy, weakness, and relative failure in addressing those prime concerns. I spell this out because I think this message remains somewhat implicit in Alan's account. Moreover, seen in this light, his intervention more obviously invites situated translations and follow-ups.

Like Alan, Ulrike's contribution is concerned with the macro picture, but she comes at the logics of the indicator game rather differently, pursuing its rhythms and their consequences. What emerges is her astute identification of a distinctive "chronopolitics" (her concept) or politics of time: she shows how it is the very specific regulations of time that constrain across a range of vectors: projects, careers, institutional budgets, etc. Speeded-up delivery, short-stories, quickturn arounds, projectification (3 year, externally-funded projects), strictly-timed careers repeatedly assessed at short intervals-these are the temporal patterns and characteristic rhythms 
she identifies. Ulrike's insight brings under scrutiny a pervasive mode of regulation which can be identified in various institutional settings, but which has been largely taken-for-granted and rarely been addressed. These temporal intensifications are, Ulrike suggests, particularly invidious as the "theatres of accountability" (Marres 2012) in academic life relentlessly proliferate. She also elaborates on Murphy's (2015) assessment that the academic world has become primarily focused on delivery, rather than discovery. Although a bit surprisingly, she does this without reference to STS's own debates about the latter as a problematic trope.

Ulrike maintains that playing the game, even "subversive compliance," is not adequate to face this pervasive escalating chronopolitics. Hence, her concern is to encourage the shifting of gears as we face the temporal (re-)structuring which she regards as the vital dimension of twentyfirst century academic evaluative culture. The tone of much of the essay is strident, as Ulrike urges resistance to the machinations of the "entrepreneurial university." Nevertheless, as noted above, she is quite open about personal episodes of enrolment in its regimes. Moreover, more than any other contributor, she explores how they capture imaginations and offer gripping imaginaries. Ulrike's despondency is infectious and, although she gestures towards ways of resisting the new temporal structures she traces, her overview of its tenacious pacing make it difficult to imagine escape.

\section{STS: Peculiarities and Resources}

There is a sense in which each of these authors is exploring the reservoir of STS resources as they seek to shake the hold of audit culture in their institutional worlds. But it is the relative newcomer to the STS in the group, Ruth Müller, who most deliberately pursues its features and distinct capacities relevant in struggles within new metric infrastructures and regulation. She lights upon the interdisciplinary roots of STS and its "emancipatory political agenda" as its most significant aspects. It is this characterization which sustains her call for STS to develop its "own" "meaningful" standards of quality.

There is much that is attractive about Ruth's proposal, not least that it works directly from the strengths of STS. It folds STS-developed perspectives on "matters of concern" (Latour 2004) and "matters of care" (la Bellacasa 2012) into the conceptualization of alternative cultures of evaluation. It also registers the range of activities that STS encompasses: not just research, but teaching, collegial support, and other forms of institutional labor. Although some of the other contributors allude to teaching (Fochler and de Rijcke 2017, 29), research and research evaluation are the main focus of this debate section. This is, in part, no doubt because some contributors are in research-only units. However, one consequence of recent research assessment regimes is that they often effectively relegate teaching to a secondary activity and sever or deny important connections between teaching and research.'

Ruth's intervention has a utopian feel to it. It builds on what she (and many others, myself included) find(s) most attractive about STS: its interdisciplinary orientations, interest in the "deeply political character of knowledge production and technology development," and the ambitions to "interfere with our technoscientific worlds critically and caringly" (Müller 2017, 86). 
Nevertheless, there are other strains within STS that are far less attractive including technocratic and scientistic inclinations that could make this difficult. Moreover, in an increasingly interdisciplinary academic world, it may not be easy or even desirable to establish "standards of our own." Indeed, in discussing the deployments of STS in the service of the natural sciences or engineering, Ruth touches upon one potential set of constraining circumstances.

\section{The Publishing Juggernaut}

Of the many threads to this assemblage that merit highlighting, the attention given to the publishing industry is especially noteworthy. A number of the contributors consider the consequences of English-language, peer-reviewed articles in international journals with high impact rankings published by a handful of commercial companies becoming the main academic currency given the indicator turn (for example, Bal 2017). However, two of the articles flesh out this trend in more detail, coming at it from two very different vantage points.

Katie Vann's (2017) analysis relates to her position as editor of ST\&HV and ESTS, as she addresses the data-centric evaluation process expressed primarily as "JIF (Journal Impact Factor) centrism" with which academic communities have increasingly become engaged and, indeed, sometimes enthralled. The problem lies not in calculation, but in centrism, she insists. She brilliantly exposes the "emperor's new clothes" character of the obsession with this evaluating mechanism, disabusing the assumption that such figures provide a reliable indication of "impact." But beyond this, she unravels the consequences of JIF centrism with reference to the political economy of the oligopolistic publishing industry (Larivière et al., 2015). This is done by showing how much (and what range of) academic labor becomes simultaneously "waste" in terms of economic reward, academic status and careers, and "surplus" which international publishing companies convert into increasing profits.

Nevertheless, she maintains that it is crucial to acknowledge what the publishing industry does provide: digital infrastructure which mediates between producers (authors) and consumers (libraries, individual subscribers) and the capacity to render unique intellectual content (articles) into standardized digital objects. The rub, as she sees it, is that the increase in scale in this industry has severe consequences for academic communities, whilst incurring limited extra cost (given economies of scale) and much potential for growing profits for publishers. For academic communities this involves increased demand for largely unrewarded labor (editing, reviewing, copy-editing, etc.) and a spiral of dependency on commercial publishers and data-generating agencies. As Katie puts it, data-deferral and data-centrism "deepen the reliance of public sector academic communities on those services and further bolster the financial gains of the companies that offer them" (Vann 2017, 106).

Katie's is one of the clearest, most succinct analyses I have encountered of the hold private publishing enterprises have on academic communities and their members and it foregrounds the compulsive dynamic of this relationship. The other great strength of her appraisal is that she pinpoints what publishers are providing that is the lever of their hold. She calls for acknowledgement that, unless or until academic communities can step into that breech, 
commercial publishers will continue to have us over a barrel. Along the way she proposes ways that open-access, non-profit journals could improve their situation.

Of course she is right, but there could also be investigations of other ways of dealing with this publishing juggernaut. For example, this might include: making the so-called "invisible work" more visible and rewarding it: demanding more of mainstream publishers (e.g. fees, support--for institutions, communities and individual scholars), tougher negotiations about their rights and controls, etc. There is the additional issue that the designation "public sector academic communities" [Katie's term, my emphasis] masques the complexity of institutional locations in increasingly privatized/commercialized (in Ulrike's term--"entrepreneurial") universities which share many of the operating principles of and, are thus unlikely to challenge, commercial publishers. In short, Katie's article should not be taken as the last word: but rather as an invigorating invitation to more critical investigation of commercial publishing and more experimentation in challenging its hold.

This brings me to another contribution to the collection which is precisely about experimentation with alternative forms of publishing within the STS community. Julien McHardy writes about a collective intervention which is oriented towards circumnavigation of the commercial/private sector publishing world: offering alternatives to its processes and practices as a vehicle for the production and dissemination of STS research and thinking. He writes as a member of the Mattering Press collective, the latter composed (originally) of earlycareer, completing or recently completed, STS PhD students, which was launched in 2012. In fact, Julien, in STS appropriate style, contends that the collective that constitutes this press extends beyond these editors to include: web developers, proof readers, copy editors, typesetters, designers, peer groups, as well as grants and donations. He takes us through the group's efforts to open-up and maintain what he calls "bewildering spaces within" the world of publishing. This is about creating the possibility of academic work that cannot be evaluated according to dominant indicator standards.

Julien's account of Mattering Press's experimentation is refreshing in a number of respects. It acknowledges the bumps on their road--that the Mattering Press collective is not charting a straight-forward alternative path. They must make concessions: such as initially publishing books by established scholars to gain credibility and resources which will enable them to produce those of new members of the community. They learn and teach us about the structures that they cannot circumvent: including the two companies that control the POD (print-on-demand) sector, one of which is the multinational giant--Amazon. Despite wrestling with such entanglements, the essay conveys an almost relentless appetite for alternative, potentially better, ways of writing, publishing, and disseminating STS research-substituting collectivity for individualization wherever possible; resistance to "playing "the system"; and generally seeking "New Songs to New Music" (McHardy 2017, 81). Ingenuity about political/intellectual resourcing animates the commentary, as Julien revisits STS principles including "non-qualculation" (Callon and Law 2005), "love," "care" (Hilary Rose, Annemarie Mol, Jeannette Pols and Vicky Singleton; Maria Puig de Bellacasa 2012), as well as drawing inspiration from Marxist philosophy (Paul LaFargue on the "right to be lazy") and art (John 
Knight). Mattering Press is clearly precarious and it is not going to stop the publishing juggernaut: but it is an STS experiment that deserves support and which could inspire other initiatives.

In their very different ways and in very different styles these two pieces expose aspects of the political economy of contemporary academic publishing and the entanglements of academia with this world. These are short, punchy interventions which underscore the need for further investigation of the publishing industry: its profitmaking, its powers for extracting free academic labor and in shaping the norms, practices and products of STS and other academic communities. They also invite experimentation to find other ways of doing and disseminating STS work.

\section{The Complication of Strategies}

After my introduction welcoming this distinctive set of STS essays, my commentary began with reflections on being implicated in the indicator game and finished with a discussion of the presentation of an explicitly alternative intervention. Nevertheless, all of these essays record and consider mixtures of individual and collective forms of both implication and contestation. Indeed, Julien ends his reflections on Mattering Press by noting its (and also ESTS's) dependence on precarious and invisible labor-the motor of neoliberal capitalism. There are clearly no neat solutions or untainted strategies.

Still, the call, the workshop outlined in the introduction (Fochler and de Rijcke 2017) and this set of essays all articulate a strong sense that STS needs strategies in response to new modes of evaluation, measurement and competition that are restructuring academic institutions, practices, and lives. I fully endorse this, as well as appreciating that this can and will be approached in a variety of ways. This collection illustrates some of these: exchanging narratives about living with and challenging performance evaluations; exploring the logics and macro patterns of academic ranking and appraisal regimes; probing the features of STS and invoking its capacities to tackle the restrictions of the indicator game; and taking-on the latter's powerful agents and corporate beneficiaries--most obviously (but perhaps not exclusively)--commercial publishers.

In encouraging these and other initiatives perhaps we could also reflect a bit on strategies themselves. They involve deliberative, collective appraisals about possible interventions. As this collection demonstrates, these are vital for STS and academia more generally. However, we might note that Julien, with reference to his alternative publishing group, observes that "good intentions do not necessarily play out right" (McHardy 2017, 79). While strategies are more than "good intentions," this caution is relevant. When pursued, strategies have consequences in complex, dynamic situations. As we know well from the theorizations of co-production and actor network theories (as well as our daily lives), such collectivities are complex, shifting, and unpredictable.

Moreover, recent STS theory has introduced more than a note of caution about human agency, thinking, and interventions as we have become more aware of the agency of "others" 
(animals, matter, etc.) and more wary of presumptions and assumptions about human powers-about human-centrism or humanism (in a broad sense). Casting a critical eye on human-centered perspectives has been a healthy antidote for those studying the often bombastic world of science and technology. Perhaps this also suggests a need for wariness about our strategizing. While this must not become a justification for political passivity, it does signal the need to be modest (without seeking to be "modest witnesses" (Haraway 1997) in the traditional mode) in devising strategies-allowing for unforeseen consequences, mindful of other worldly agents, and of the unpredictability of the outcome of our interventions.

There are discernible notes of caution throughout this collection: about co-option and implicatedness; about diversity in locations (amongst departments or research units; universities; national settings, etc.) and in positionings (with particular sensitivities about those of early career and potential future researchers). This bodes well for the prospects of further "evaluative inquiry" in response to the invitation the editors of this issue have extended to the STS community.

\section{Author Biography}

Maureen McNeil is Professor Emerita of Sociology, Lancaster University. During her forty-year career she has been a model of generous mentorship and collaborative engagement in the service of the collective flourishing of both her students and her colleagues. She also has addressed wider systemic arrangements that undervalue such work. Her teaching is based in feminist pedagogy and practice, placing emphasis on critical questioning in an atmosphere of open discussion and respectful debate. In 2015 she received the Mentoring Award of the Society for Social Studies of Science.

\section{References}

Bal, R. 2017. "Playing the Indicator Game: Reflections on Strategies to Position a Group in a Multidisciplinary Environment." Engaging Science, Technology, and Society 3: 41-52. DOI:10.17351/ ests2016.111.

Callon, M. and J. Law. 2005. "On qualculation, agency, and otherness." Environment and Planning D: Society and Space, 23(5): 717-733.

Felt, U. 2017. "Under the Shadow of Time: Where Indicators and Academic Values Meet." Engaging Science, Technology, and Society 3: 53-63. DOI:10.17351/ ests2016.109.

Fochler, M. and S. de Rijcke. 2017. "Implicated in the Indicator Game? An Experimental Debate." Engaging Science, Technology, and Society 3: 21-40. DOI:10.17351/ests2017.108.

Haraway, D. J. 1997. Modest_Witness@Second_Millennium. Femaleman@_Meets_Oncomouse ${ }^{\mathrm{TM}}$ : Feminism and Technoscience. London and New York: Routledge.

Irwin, A. 2017. "If the Indicator Game is the Answer, then What is the Question?" Engaging Science, Technology, and Society 3: 64-72. DOI:10.17351/ests2017.110.

la Bellacasa, de, M. P. 2012. "Nothing comes without its world: thinking with care." The 
Sociological Review, 60(2): 197-216. http:/ / doi.org/10.1111/j.1467-954X.2012.02070.x.

Lafargue, P. 1883. The Right To Be Lazy and Other Studies. London: Charles Kerr and Co. Online Version: Lafargue Internet Archive. Accessed June 222016. https: / / www.marxists.org/archive/lafargue/1883/lazy/

Larivière V., S. Haustein, and P. Mongeon (2015) "The Oligopoly of Academic Publishers in the Digital Era." PLOS ONE 10(6), 1-15. doi:10.1371/journal.pone.0127502.

Marres, N. 2012. Material Participation: Technology, the Environment and Everyday Publics: Palgrave Macmillan.

McHardy, J. 2017. "Like Cream: Valuing the Invaluable." Engaging Science, Technology, and Society 3: 73-83. DOI:10.17351/ ests2017.116.

Müller, R. 2017. "Crafting a Career in STS: Meaning Making, Assessment, and Interdisciplinary Engagement." Engaging Science, Technology, and Society 3: 84-91. DOI:10.17351/ ests2017.112.

Murphy, P. 2015. "Discovery and delivery: time schemas and the bureaucratic university." In Universitites in the Flux of Time: An Exploration of Time and Temporality in University Life, edited by Paul Gibbs, Oili-Helena Ylijoki, Carolina Guzmán-Valenzuela and Roland Barnett, 137-153. London: Routledge.

Strathern, M. 1997. "Improving ratings': Audit in the British University System." European Review 5 (03): 305-321.

Vann, K. 2017. "Surplus and Indicator." Engaging Science, Technology, and Society 3: 92-107. DOI:10.17351/ ests2016.113.

Wouters, P. 2017 "Bridging the Evaluation Gap." Engaging Science, Technology, and Society 3: 108118. DOI:10.17351/ests2017.115.

"This has led, in the UK at least, to the phenomena of "teaching-only" contracts. However, this is not to say that teaching has not itself been subjected to quantitative assessments and rankings as the editors of this collection indicate (Fochler and de Rijcke 2017). 